\title{
Common single nucleotide polymorphisms in the FNDC5 gene and serum irisin levels in acute myocardial infarction
}

\author{
Ebru Önalan Etem* (iD, Özge Diş* (iD), Ahmet Tektemur* (iD, Hasan Korkmaz** (iD, İlay Buran Kavuran* (i) \\ Departments of *Medical Biology, and **Cardiology, Faculty of Medicine, Firat University; Elazı̆̆-Turkey
}

\section{ABSTRACT}

Objective: Acute myocardial infarction (AMI) is the most common type of coronary artery disease. The irisin hormone encoded by the fibronectin type III domain-containing protein-5 (FNDC5) gene is synthesized in muscle, heart, and fat tissues. The present study aims to investigate serum irisin concentrations and FNDC5 genetic variants in patients with AMI through comparison with controls.

Methods: This study included 225 patients with AMI and 225 healthy subjects. Blood samples were obtained from patients during the first $1-24$ hours after AMI. Serum irisin concentration was measured with enzyme-linked immunosorbent assay (ELISA). The variants of rs16835198, rs3480, and rs726344 in the FNDC5 gene were genotyped with real time polymerase chain reaction (RT-PCR).

Results: Compared with control serum irisin concentrations were significantly lower in patients with AMI. Serum irisin concentrations of patients with AMI showed a significant and gradual decrease from 6 hours up to 24 hours $(p<0.05)$. There were no significant differences between the patient and control groups based on genotype and allele frequencies of rs16835198, rs3480, and rs726344 in the FNDC5 gene $(p>0.05)$. However, the frequency of the TT genotype in male patients with AMI (6.4\%) was significantly lower compared with control male subjects (16.2\%). In addition, the GGT haplotype was identified as the protective haplotype against the risk of $A M I$ ( $p<0.001$; odds ratio=0.107). Conclusions: The findings of the study suggest that serum irisin concentration could serve as a novel biological marker for the early diagnosis of AMI. Keywords: acute myocardial infarction, single nucleotide polymorphism, haplotype analysis, irisin, FNDC5

Cite this article as: Önalan Etem E, Diş Ö, Tektemur A, Korkmaz H, Buran Kavuran I. Common single nucleotide polymorphisms in the FNDC5 gene and serum irisin levels in acute myocardial infarction. Anatol J Cardiol 2021; 25: 528-35.

\section{Introduction}

Acute myocardial infarction (AMI) is currently one of the most important reasons of mortality and morbidity in many countries (1). Detection of familial clustering of early-onset myocardial infarction by prospective studies has confirmed that there is a complex and multifactorial genetic predisposition to the disease in addition to the traditional known risk factors. In assessing the genetic risks for the development of $\mathrm{AMI}$ and in determining the biomarkers for disease detection, the genes encoding factors released into the blood during AMI are used (2).

In 2012, Boström et al. (3) discovered a new hormone named irisin, which is released by the skeletal muscle after systematic exercise with the potential to protect the individual from metabolic diseases. Irisin is a myokine with 112 amino acids that is produced upon the cleavage of the plasma membrane protein fibronectin type III domain-containing protein 5 (FNDC5) and enters circulation (3). This hormone is also a thermogenic myokine that converts white adipose tissue (WAT) into brown adipose tissue (BAT) in response to the activation of peroxisome proliferator-activated receptor-gamma coactivator- $1 \alpha$ (PGC$1 \alpha)$, leading to energy consumption and contributing to muscleadipose tissue cross-talk $(3,4)$. Although irisin is secreted mostly from skeletal muscle after exercise, it is also expressed by the heart, tongue, rectum, pancreas, liver, adipose tissue, nervous system, placenta, and ovary, with particularly high levels in the heart (2). The secretion of irisin by the heart muscle led to the hypothesis that it could play a role in the etiopathogenesis of cardiovascular diseases and could be a biomarker for AMI. There is a limited number of studies that analyzed serum or

Address for Correspondence: Dr. İlay Buran Kavuran, Fırat Üniversitesi Tıp Fakültesi, Tıbbi Biyoloji Anabilim Dalı, Elazığ-Türkiye

Phone: +90 42423700 00/4646 E-mail: ilayburan@hotmail.com/iburan@firat.edu.tr

Accepted Date: 08.02.2021 Available Online Date: 10.07.2021

(C) Copyright 2021 by Turkish Society of Cardiology - Available online at www.anatoljcardiol.com DOI:10.5152/AnatolJCardiol.2021.36214 


\section{HIGHLIGHTS}

- The decreased TT genotype of the rs16835198 G>T SNP was associated with higher risk in AMI development in male patients.

- The GGT-specific haplotype combinations of rs3480, rs726344, and rs168371198 can affect AMI risk.

- Irisin levels were analyzed for the first time in such a large group of patients with AMI, and irisin levels gradually decreased in the first 24 hours.

heart tissue irisin levels in human and animal MI models. Furthermore, serum irisin levels were found to decrease after $\mathrm{AMI}$ and to be positively correlated with troponin I, creatine kinase myocardial band (CK-MB), and creatine kinase (CK) $(5,6)$.

The FNDC5 gene that contains six exons and five introns is located in chromosome $1 \mathrm{p} 35.1$ (7). Among the introns of the gene, 5 common single nucleotide polymorphisms (SNPs) have been defined (8). The associations of the common variants of the FNDC5 gene and serum irisin levels with obesity, anorexia nervosa, insulin sensitivity, nonalcoholic fatty liver disease, and chronic kidney disease have been previously investigated (7-12). The tested FNDC5 rs16835198, rs3480, and rs726344 SNPs were not related to serum irisin levels in some studies but related in others $(13,14)$. The rs 16835198, rs3480, and rs726344 SNPs do not lead to amino acid changes in the irisin protein, which is a FNDC5 gene product. The variant A allele of the rs726344 SNP and the variant T allele of the rs16835198 SNP have higher luciferase activity when compared with the wild allele (9). The rs16835198 SNP is localized in the $3^{\prime}$ end region of the gene. Nucleotide changes in the 3 -untranslated region of a gene might influence the regulation of gene expression. As in rs726344, intronic genomic variants can alter gene expression and thus affect the phenotype by altering the binding of transcription factors, alternative mRNA clipping, and mRNA stability $(14,15)$. The rs $3480 \mathrm{~A}$ allele is also associated with a higher FNDC5 mRNA break when compared with the G allele, which is thought as the binding of miR-135a-5P (16-19). The main reason for choosing these three SNPs among the known FNDC5 gene polymorphisms in the present study is that they are both associated with metabolic phenotypes and fibrosis, and the variant genotypes have higher luciferase activity (13-15).

Because FNDC5 is expressed in the heart, a relationship has been determined between AMI and reduced irisin levels, and the present study aims to investigate the serum irisin levels and to determine rs 16835198, rs726344, and rs3480 genetic variants in the patients with AMI compared with controls, and to assess the utility of irisin as a biomarker in AMI patients.

\section{Methods}

\section{Study design}

This is a prospective case-control study. The present study included 225 patients diagnosed with $\mathrm{Ml}$ and admitted to the
Department of Cardiology at Firat University Hospital and 225 healthy individuals as controls, who were comparable to the patient group in terms of age and sex. All participants were provided verbal information on genetic tests and $\mathrm{Ml}$. A total of 450 subjects who agreed to participate were informed about the details of the study, and informed consent forms were obtained. Ethics approval for the study was obtained by Firat University, Faculty of Medicine Local Ethics Committee (14.10.2014, Decision No: 17/07). Acute MI was diagnosed via surface electrocardiogram (ECG) and/or cardiac biomarkers obtained during admission to the intensive care unit in the presence of clinical symptoms and findings. The acute AMI criteria included chest pain lasting at least 20 minutes, at least $\geq 1 \mathrm{~mm}$ ST elevation in at least 2 adjacent derivations on ECG, and admittance to the coronary care unit 12 hours after the onset of pain. Thirty-four non ST-elevation myocardial infarction (NSTEMI) patients with proven myocardial damage by elevation of damage markers were also included in the present study. Blood was collected within the first 24 hours after AMI. Individuals with chest pain for over 12 hours, patients over 80 years of age, patients diagnosed with chronic renal failure (serum creatinine $>2.5$ ), chronic liver failure, morbid obesity, and clinical heart failure (Killip II/III), and patients with findings indicating cardiogenic shock, malignancies, and chronic inflammatory diseases that could lead to noncardiac systemic inflammation were excluded. Anamnesis was obtained to determine whether the patients had risk factors (hypertension, diabetes, smoking, alcohol), family history, and ischemic stroke.

\section{Sample collection}

Blood samples of the participants were taken into tubes with aprotinin, as part of a routine procedure within the first 1-24 hours. Three-ml blood samples were centrifuged at $3000 \mathrm{rpm}$ for $10 \mathrm{~min}$ utes to obtain sera. Pellets remaining at the bottom and sera at the top of the sample tube were stored at $-80^{\circ} \mathrm{C}$ until used for DNA isolation and enzyme-linked immunosorbent assay (ELISA) analysis.

\section{Real Time PCR (RT-PCR) analysis}

DNA was isolated using the Wizard Genomic DNA Purification Kit (CAT \# A1120, Promega, Madison, WI, USA) from 2-3 ml peripheral venous blood pellets. DNA concentrations were diluted to 1-10 ng. rs3480 (Cat No: C-8822841-10, Applied Biosystems, Foster City, CA, USA), rs726344 (C-927694-10, Applied Biosystems), and rs16835198 (C-34204885-10, Applied Biosystems) SNPs were studied with TaqMan probes (Applied Biosystems) and Taqman genotyping master mix (Catalog number: 4371355) on the ABI 7500 Fast Real Time System (Applied Biosystems). After PCR, homozygous mutant, heterozygous, and homozygous normal genotypes were determined based on the allele 1 and allele 2 separation using the software available on the device.

\section{Biochemical analyses}

Irisin levels were studied with the ELISA method (Catalog No: YLA1361HU, YL Biont, Shanghai, China). Measurements were conducted with a Biotek Epoch microplate spectrophotometer (EL X 800 / Bio-Tek Instruments, Winooski, VT, USA). 


\section{Statistical analyses}

All descriptive and inferential statistical analyses were performed with SPSS version 22.0 (IBM Corp., Chicago, IL, USA). Data are presented in counts, percentages, mean, and standard deviation values. The Kolmogorov-Smirnov test was conducted to determine whether the data were distributed normally. The deviation from Hardy-Weinberg equilibrium (HWE) were verified using Chi-square $\left(\chi^{2}\right)$ test. Chi-square tests were also used to make a comparison between groups, for the analysis of the association between two qualitative variables, and allele and genotype frequencies. Haplotypes constructed from a combination of the three FNDC5 SNPs were analyzed using the SHesis program (http://analysis.bio-x.cn/myAnalysis.php) (20). This program was also used to compare FNDC5 haplotype frequencies between AMI patients and control subjects. The magnitude of the association between FNDC5 SNPs and AMI was expressed by odds ratio (OR) and $95 \%$ confidence interval (CI). Demographics, clinical, and laboratory data were compared between groups by Student's t test, chi-square test, and oneway ANOVA test (F). Correlation analyses were performed by Pearson's correlation analysis. ORs and $p$ values of the variables were calculated at $95 \%$ confidence level. $P<0.05$ was considered statistically significant.

\section{Results}

Relationship between demographic, clinical, and biochemical parameters and irisin levels in patients with AMI

Demographic, clinical, and biochemical properties of AMI and control groups are presented in Table 1. T-test results showed that serum CK-MB and troponin T levels significantly increased, and serum irisin levels significantly decreased in AMI patients when compared with the control subjects $(p=0.001$, $p=0.001$, and $p=0.001$, respectively). Serum troponin $T$ values were significantly higher in females than in males $(p=0.003)$, but there were no significant differences between CK-MB, lactate dehydrogenase (LDH), and irisin levels based on gender in the patient group $(p=0.265, p=0.470, p=0.188$, respectively), according to the $t$ test. Comparison of the irisin levels of the patient and control groups based on gender demonstrated that irisin levels were only significantly higher in males compared with females in the control group $(4.45 \pm 2.34 \mathrm{ng} / \mathrm{ml}$ for females and $3.39 \pm 1.86$ $\mathrm{ng} / \mathrm{ml}$ for males the serum irisin concentration; $\mathrm{p}=0.023$ ), with no such gender differences detected in the patient group.

The serum irisin level was positively correlated with body mass index (BMI), weight, troponin $\mathrm{T}$ in the patient group according to Pearson's correlation analysis $(p<0.001, r=0.383$; $p<0.001, r=0.898 ; p<0.001, r=0.904$, respectively). There was also a strong negative correlation between age, CK-MB, pulse blood pressure, systolic blood pressure, diastolic blood pressure, and serum irisin level in the patient group according to Pearson's correlation analysis $(p<0.001, r=-0.626 ; p<0.001, r=-0.245$; $p=0.001, r=-0.235 ; p<0.001, r=-0.450 ; p<0.001, r=-0.563$, respectively). Serum irisin concentrations [ $1-3 \mathrm{~h}(\mathrm{n}=46): 4.75 \pm 0.50 \mathrm{ng} / \mathrm{ml}$; $3-6 \mathrm{~h}(\mathrm{n}=48): 3.82 \pm 0.37 ; 6-12 \mathrm{~h}(\mathrm{n}=60): 3.47 \pm 0.24 \mathrm{ng} / \mathrm{ml} ; 12-18 \mathrm{~h}$
Table 1. Demographic, clinical, and biochemical characteristics of patients with AMI

\begin{tabular}{|c|c|c|c|}
\hline Variables & $\begin{array}{c}\text { Control }(\mathrm{n}=225) \\
\text { (Mean } \pm \text { SD) }\end{array}$ & $\begin{array}{l}\text { AMI }(n=225) \\
(M e a n \pm \text { SD) }\end{array}$ & $\boldsymbol{P}$-value \\
\hline Age (years) & $63.83 \pm 12.25$ & $64.11 \pm 12.28$ & 0.834 \\
\hline $\operatorname{Sex}(M / W)$ & $148 / 77$ & $156 / 69$ & 0.421 \\
\hline Hypertension (\%) & - & (54.2) 122 & - \\
\hline Diabetes (\%) & - & (44) 99 & - \\
\hline $\begin{array}{l}\text { Body mass index } \\
\left(\mathrm{kg} / \mathrm{m}^{2}\right)\end{array}$ & $24.4 \pm 4.82$ & $26.46 \pm 4.76$ & 0.158 \\
\hline Smoking (\%) & (47.6) 107 & (44.4) 100 & 0.508 \\
\hline Family history (\%) & - & (67.6) 152 & - \\
\hline CK-MB (ng/mL) & $27.45 \pm 25.68$ & $69.01 \pm 61.38$ & $0.001 *$ \\
\hline Troponin-T (ng/mL) & $0.7 \pm 1.74$ & $11.94 \pm 11.05$ & $0.001 *$ \\
\hline $\begin{array}{l}\text { Systolic blood } \\
\text { pressure }(\mathrm{mm} \mathrm{Hg})\end{array}$ & $118.2 \pm 21$ & $117.7 \pm 26$ & 0.724 \\
\hline $\begin{array}{l}\text { Diastolic blood } \\
\text { pressure }(\mathrm{mm} \mathrm{Hg})\end{array}$ & $76 \pm 9$ & $78.16 \pm 16.18$ & 0.131 \\
\hline $\mathrm{LDH}(\mathrm{mg} / \mathrm{dL})$ & $285.4 \pm 90.57$ & $334.24 \pm 146.1$ & 0.257 \\
\hline \multicolumn{4}{|l|}{$\begin{array}{l}\text { Myocardial infarction } \\
\text { type }(\%)\end{array}$} \\
\hline A. inferior & & (48.9) 110 & \\
\hline A. anterior & & (36) 81 & \\
\hline NSTEMI & - & $(15.1) 34$ & - \\
\hline EF & - & $46.19 \pm 10.44$ & - \\
\hline Irisin (ng/mL) & $4.02 \pm 2.22$ & $3.14 \pm 2.05$ & $0.001 *$ \\
\hline \multicolumn{4}{|c|}{$\begin{array}{l}\text { *: } P<0.001 \text {. The values are presented as mean } \pm S D \text {. Mann-Whitney } \mathrm{U} \text { and chi-square } \\
\text { tests were performed. Statistically significant values were marked in bold. } \\
\text { AMI - acute myocardial infarction; SD - standard deviation; EF - ejection fraction; } \\
\text { NSTEMI - non-ST-elevation myocardial infarction; LDH - lactate dehydrogenase; CK-MB } \\
\text { - creatine kinase myocardial band }\end{array}$} \\
\hline
\end{tabular}

$(\mathrm{n}=49): 2.28 \pm 0.26 ; 18-24 \mathrm{~h}(\mathrm{n}=22): 1.38 \pm 0.14 \mathrm{ng} / \mathrm{ml}$ of AMI patients showed a significant and gradual decrease from $6 \mathrm{~h}$ up to $24 \mathrm{~h}$ $(p<0.000, F=9.050$, Fig. 1).

Systolic blood pressure values were significantly higher in patients with hypertension (HT) than in those without hypertension (non-HT) $(p=0.048)$, but no significant difference was determined between patients with and without $\mathrm{HT}$ in terms of troponin T, CK-MB, LDH, and diastolic blood pressure according to the $t$ test $(p=0.151, p=0.417, p=0.585, p=0.704$, respectively). The same comparison revealed that irisin levels were also significantly lower in individuals with HT group when compared with control and non-HT groups ( $p=0.001, p=0.018$, respectively). There was no significant difference in irisin levels between control and nonHT $(p=0.737)$. It was determined that the irisin levels of the patients with diabetes mellitus (DM) decreased significantly, but the irisin levels of the patients without diabetes (non-DM) did not change significantly compared with the control $(p=0.047, p=0.065$, respectively). There was no significant difference in irisin levels between DM and non-DM groups ( $p=0.944$ ) (Fig. 2). 


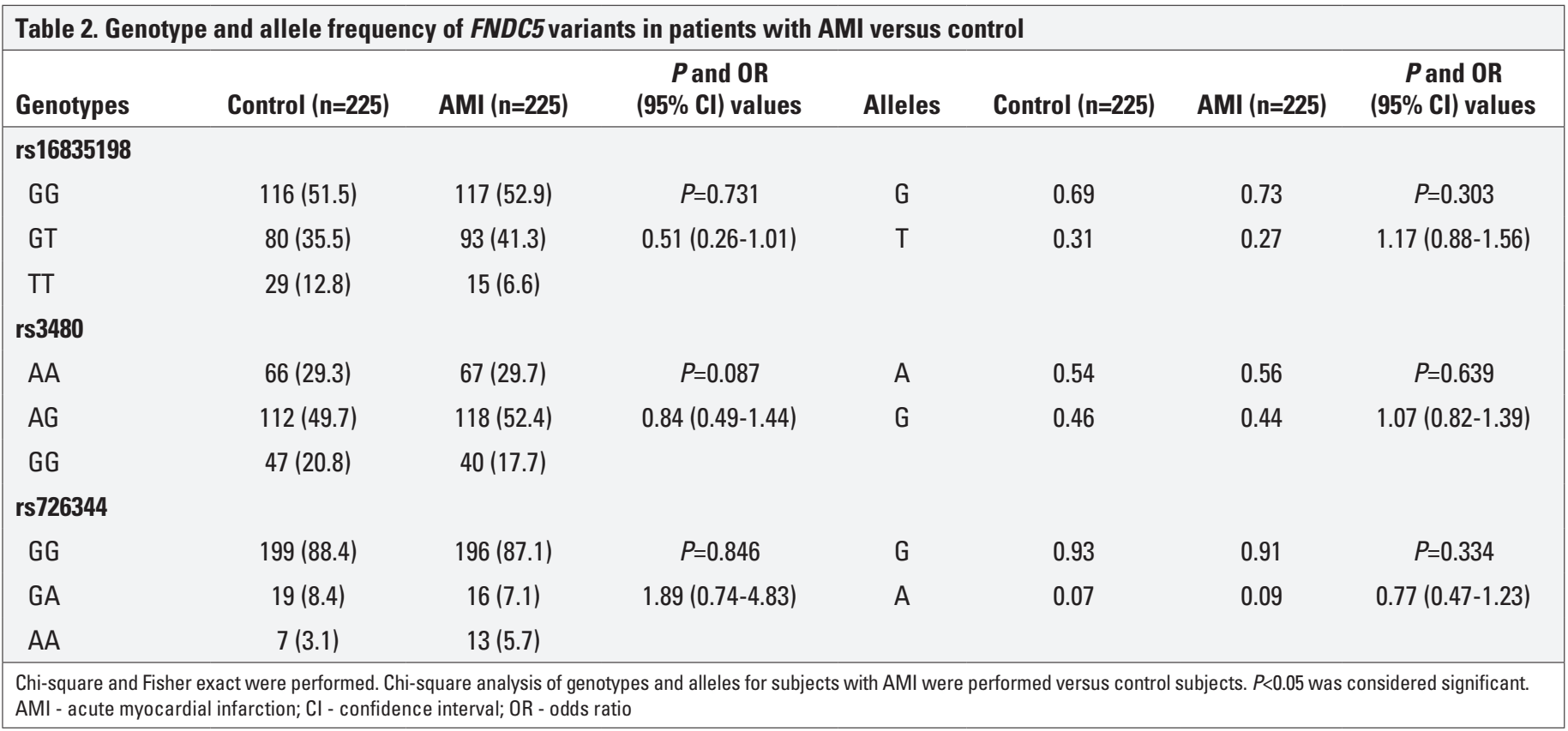

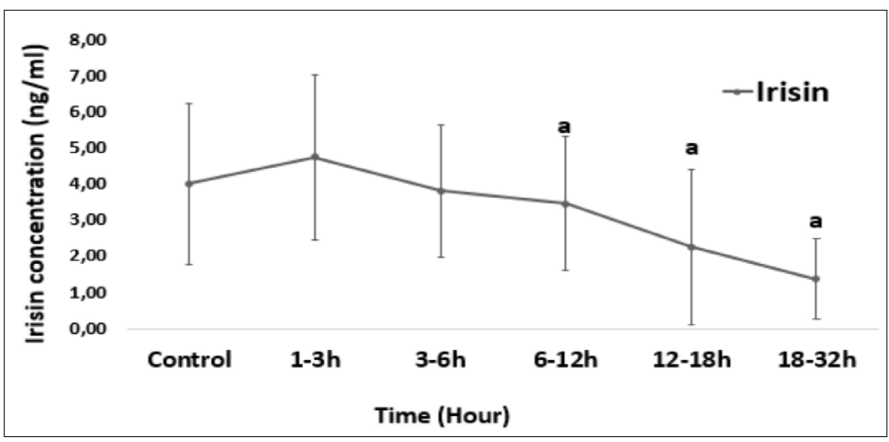

Figure 1. Circulating irisin levels are gradually decreased in 24 hours after acute myocardial infarction. Values are expressed in $\mathrm{ng} / \mathrm{ml}$ for circulating irisin. "a" letter above the line indicate statistical differences (t test, a value of $P<0.05$ was considered statistically significant). Data are shown as mean \pm SD

a: $P<0.05$ vs. control subjects.

\section{Genotype and allele distributions}

The distribution of genotype and allele frequencies of FNDC5 gene variants in AMI and control groups are presented in Table 2. All analyzed SNPs were in the Hardy-Weinberg equilibrium in AMI and control subjects. It was found that there were no significant differences between the patient and control groups in terms of the rs16835198, rs3480, and rs7266344 genotype distributions ( $p=0.731$, $p=0.087, p=0.846$, respectively) and allele frequencies $(p=0.303$, $\mathrm{p}=0.639, \mathrm{p}=0.334$, respectively) of the three analyzed SNPs (Table 2). In the comparison of allele frequencies and the genotype distribution of FNDC5 rs16835198, rs3480, and rs7266344 SNPs across genders, it was found that the TT genotype of the rs16835198 G>T SNP was statistically higher in control male subjects than in male patients with $A M I[p=0.037,0 R=0.69(0.49-0.97)$ for genotypes and $\mathrm{p}=0.025, \mathrm{OR}=0.34(0.15-0.77)$ for alleles]. Genotype distribution of rs $16835198 \mathrm{G}>\mathrm{T}$ SNP was determined in 78 patients with $\mathrm{AMI}(50 \%)$ for wild type genotype, 68 (43.5\%) for heterozygote genotype, and

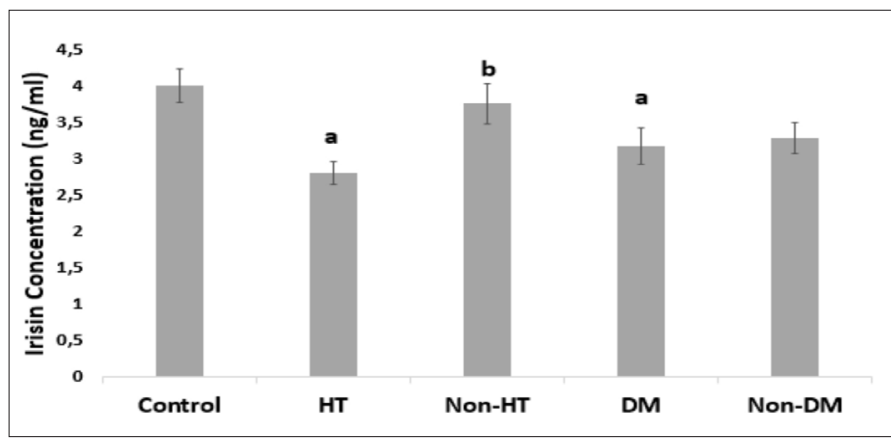

Figure 2. Serum irisin levels in patients with and without diabetes and hypertension (HT). Values are expressed in $\mathrm{ng} / \mathrm{ml}$ for circulating irisin. Letters above the line indicate statistical differences (t test, a value of $P<0.05$ was considered statistically significant). Data are shown as mean \pm SEM

a: $P<0.05$ vs. control subjects. b: $P<0.05$ vs. subjects with $\mathrm{HT}$

$10(6.41 \%)$ for polymorphic genotype. Furthermore, for rs 16835198 G>T SNP, 65 of control subjects (43.9\%) were homozygous for wild type genotype, $59(43.5 \%)$ were heterozygous, and only $24(16.2 \%)$ were polymorphic homozygous. Allele frequencies in patients with AMI were 0.72 for the wild $G$ allele and 0.28 for polymorphic $T$ allele. The frequency of wild-type allele, was 0.64 and that of the polymorphic allele was 0.36 , which represented $16.2 \%$ of the examined control subjects. The frequency of the TT genotype in male patients with AMI $(6.4 \%)$ was significantly lower compared with control male subjects (16.2\%). Comparison of the genotype distributions and allele frequencies of the three analyzed rs16835198, rs3480, and rs7266344 SNPs in the FNDC5 gene between AMI patients with diabetes $(\mathrm{p}=0.137, \mathrm{p}=0.060, \mathrm{p}=0.179$ for genotypes; $p=0.303, p=0.080, p=0.204$ for alleles, respectively) or hypertension $(p=0.923, p=0.572, p=0.163$ for genotypes; $p=0.731, p=0.576, p=0.197$ for alleles, respectively) and the control group revealed no significant differences. 


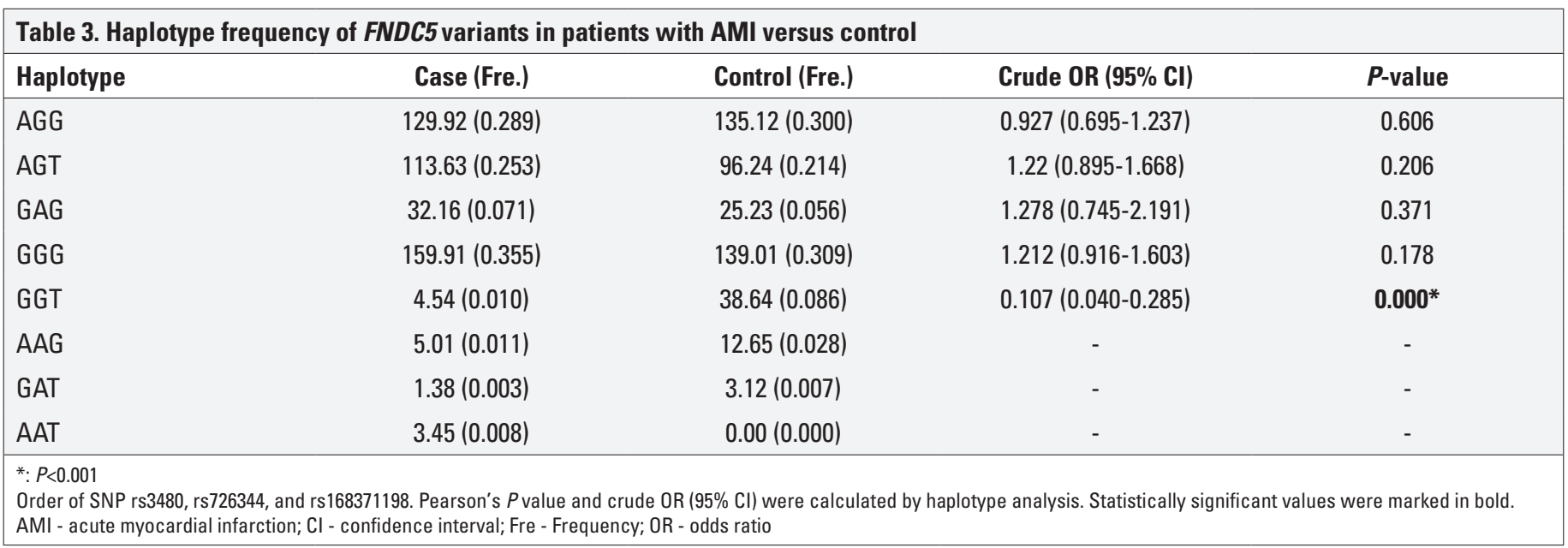

\section{Relationship between demographic and biochemical} parameters and genotypes in patients with AMI

These correlation analyses were performed only in AMI group. There was no correlation between genotype distributions of rs16835198 and rs7266344 and the patients' BMI, systolic blood pressure $(\mathrm{mm} \mathrm{Hg})$, diastolic blood pressure $(\mathrm{mm} \mathrm{Hg})$, troponin T, CK-MB, LDH, diastolic left ventricular diameter, diastolic right ventricular diameter, ejection fraction (EF), and irisin $(p=0.729$ and $p=0.815, p=0.602$ and $p=0.865, p=0.203$ and $p=0.892$, $p=0.526$ and $p=0.123, p=0.611$ and $p=0.859, p=0.870$ and $p=0.649$, $\mathrm{p}=0.981$ and $\mathrm{p}=0.214, \mathrm{p}=0.142$ and $\mathrm{p}=0.991, \mathrm{p}=0.910$ and $\mathrm{p}=0.368$, $\mathrm{p}=0.599$ and 0.141 , respectively) We did not detect any significant correlation between rs3480 SNP and BMI, systolic blood pressure $(\mathrm{mm} \mathrm{Hg})$, troponin T, CK-MB, LDH, EF, and irisin $(p=0.712, p=0.089, p=0.239, p=0.679, p=0.547, p=0.256, p=0.925$ ， respectively). However, diastolic blood pressure was lower in the rs3480 heterozygote genotype when compared with variant and wild genotypes $(p=0.020, F=3.49 ; A A=85.15 \pm 17.12 \mathrm{~mm} \mathrm{Hg}$, $A G=73.88 \pm 12.93 \mathrm{~mm} \mathrm{Hg}, \mathrm{GG}=84.20 \pm 20.78 \mathrm{~mm} \mathrm{Hg}$ ). Diastolic left ventricular diameters increased in the rs3480 polymorphic and heterozygote genotypes compared with the wild-type genotype, according to the one-way ANOVA test $(\mathrm{p}=0.041, \mathrm{~F}=2.87$; $A A=46.61 \pm 6.07 \mathrm{~mm}, A G=50.71 \pm 7.81 \mathrm{~mm}$ and $\mathrm{GG}=49.90 \pm 7.55 \mathrm{~mm}$ ). Diastolic right ventricular diameters increased in the rs3480 heterozygote and polymorphic genotypes compared with the wild-type genotype, according to the one-way ANOVA test $(p=0.008, \quad F=4.17 ; \quad A A=21.57 \pm 2.51 \mathrm{~mm}, \quad A G=23.41 \pm 2.65 \mathrm{~mm}$, $\mathrm{GG}=24.30 \pm 3.97 \mathrm{~mm}$ ).

\section{Haplotype distributions}

The distribution frequencies of rs3480, rs726344, and rs168371198 GGT haplotypes were significantly different in patients with compared with control individuals. Notably, this haplotype was significantly correlated with a reduced AMI risk ( $p<0.001$, crude $0 \mathrm{R}=0.107 ; 95 \% \mathrm{Cl}: 0.040-0.285$ ). However, no such differences were determined in the AMI group for the other analyzed haplotypes (Table 3).

\section{Discussion}

A new thermogenic uncoupling protein, irisin is a new player in energy regulation and abundantly expressed in skeletal and cardiac muscle $(8,9)$. Many metabolic and cardiovascular diseases are related to low serum irisin levels (6). Many studies have demonstrated no association between genders, age, and serum irisin level (21). In consistence with the findings of the present study, Anastasilakis et al. (22) reported that serum irisin level was significantly lower in males than females. According to the analysis of studies in the literature investigating the association between various types of heart diseases and serum irisin levels in humans and animals, in general, serum irisin levels significantly decreased in cardiovascular diseases (CVD), consistent with the findings of the present study (23-29). In addition, a study reported that serum irisin levels were significantly lower in patients with major cardiovascular problems, stable coronary artery disease (CAD), or (MI) (23). An association with adverse cardiovascular outcomes and the increased irisin concentrations was found in the patients with ST-elevation MI (STEMI) (24). The decreased irisin levels predict the severity of stable coronary artery disease and a higher Synergy Between Percutaneous Coronary Intervention With Taxus and Cardiac Surgery (SYNTAX) score (25). Another study found that serum and saliva irisin levels decreased gradually in the first 48 hours in patients with AMI when compared with controls and then showed an increase at 72 hours (6). The same study also reported that there was a correlation between serum and salivary irisin levels and troponin I, CK-MB, and CK. They suggested that irisin could be a new diagnostic marker during the first 48 hours in patients with AMI (6). In a rat model of isoproterenol (ISO)induced $\mathrm{MI}$, it was determined that serum irisin levels significantly decreased within the first 6 hours (26), and cardiac tissue significantly increased on the second week after MI (5). In consistence with these studies, the present study also determined a positive correlation between serum irisin levels and troponin T and a negative correlation between irisin and CK-MB. The most important question regarding the post-AMI period is 
why serum irisin levels show a decrease rather than an increase. In the myocardium, energy is stored in the form of adenosine triphosphate (ATP) or creatine phosphate (CP). AMI is a condition associated with high energy demand and severe oxygen deprivation, and most of the ATP and CP stores are consumed within the first 30 minutes after ischemia (27). In the case that large amounts of irisin are rapidly released into the circulation during ischemia, the ischemic heart would experience sudden ATP depletion, which would worsen the ischemic condition. If the tissues do not respond to this condition, the development of necrosis in the ischemic site will be accelerated by the greater energy loss and heat production that will occur (28). Irisin synthesized in the myocardium in the heart is rapidly released into the circulation from the ischemic heart, similarly to troponin and CK-MB. Demonstrating a negative correlation, irisin gradually decreases in the ischemic heart during the first 48 hours, whereas CK, CK-MB, and troponin gradually increase during the first 12 hours. We reasoned that the gradual decrease in the serum irisin levels of patients within the first 1-24 hours after AMI alleviated the ATP loss during $\mathrm{MI}$ and facilitated the provision of the cellular energy demand. However, the potential mechanisms underlying the decrease in irisin after AMI have not yet been fully elucidated. One of these mechanisms is thought to involve miRNAs such as miR-34a, which bind directly to the FNDC5 mRNA. Serum miR-34a levels increased in AMI patients and rats with AMI (29), and the suppression of the mir-34 family protected the heart against pathological cardiac remodeling and to improve its functioning (30). One of the molecular mechanisms underlying the decrease in irisin subsequent to $A M I$ is thought to be the rapid increase in miRNAs such as miR-34a after AMI, which abrogates the transcription of FNDC5 mRNA and accelerates its cleavage.

Can irisin serve as an ideal biomarker for AMI? An ideal biomarker should possess certain characteristics (31). Currently, although relevant human and animal clinical experiments are scarce, the existing studies suggest that irisin decreases within the first 48 hours after AMI (6). Although our study reports the first 24 hours, it is clear that irisin decreased after AMI and that this decrease occurred in a gradual pattern, in congruence with the previous studies. Moreover, limiting the ability of the irisin to be a biomarker, the decreased irisin levels were also shown to be associated with chronic renal disease (32), the severity of CAD (25), breast cancer (33), and colorectal cancers (34). In our study, irisin was demonstrated to have a negative correlation with CK-MB and a positive correlation with troponin T. Another finding of our study that will limit the feature of irisin being a biomarker is that irisin levels did not change significantly in the non-DM and non-HT patient groups with AMI compared with the control. In addition, irisin levels showed a decrease, in contrast with the markers that are considered the gold standard in AMI such as CK, CK-MB, LDH, aspartate aminotransferase (AST), and troponin I and T. Because of this property, irisin is thought to have a potential utility in the diagnosis and follow-up of AMI. However, before irisin can be used as a diagnostic marker for $\mathrm{AMI}$, certain ambiguities and questions must be clarified.
Among these are the cut-off values of irisin, its optimum receiver operating characteristic (ROC) curve values versus cTnT, cTnl, and its sensitivity and specificity. Although irisin is a strong candidate biomarker for AMI, whether or not it can be utilized as a biomarker will be determined by large-scale studies that will be able to obtain these data.

Consistent with our study, a meta-analysis including 23 studies found that serum irisin levels were lower in patients with type II diabetes (35). In the present study, serum irisin levels were also significantly lower in the subjects with hypertension. Whereas central (intraventricular) irisin administration increased blood pressure and cardiac contractility, intravenous administration of irisin decreased the blood pressure in controls and spontaneously hypertensive rats (35). In the rat experiment mentioned above, the fact that peripheral irisin administration reduced blood pressure was consistent with the decreased hypertension in hypertensive patients. Therefore, measurement of circulating irisin levels in subjects with hypertensive may provide new insight into the pathology of these conditions.

There is a wide variance in the prevalence of the rs 16835198 SNP G allele (0.673) and T allele (0.326) (ranging between 0.02 and 0.44 worldwide, for minor allele frequency), the rs3480 SNP $\mathrm{G}$ allele (0.410) and A allele (0.589) (ranging between 0.23 and 0.54 worldwide, for minor allele frequency), and the rs726344 SNP G allele (0.862) and A allele (0.138) (ranging between 0 and 0.38 worldwide, for minor allele frequency) in different populations globally. The MAF frequencies for rs 16835198 , rs3480, and rs726344 were determined as $0.31,0.46$, and 0.07 , respectively, in the control group of the present study. MAF frequencies in the current study are comparable to those determined in Asian and European populations. Although the FNDC5 allelic variants in humans have been implicated in diabetes, obesity, aging, nonalcoholic fatty liver disease, preterm birth, and exercise $(9,14-19$, 36), the studies for the association and haplotype analysis of the FNDC5 gene in subjects with AMI is very limited. Only Badr et al. (37) conducted a small study including $100 \mathrm{AMI}$ and 100 control Egyptian subjects for the analysis of FNDC5 gene polymorphism. Contrary to our study, they reported that troponin I and triglyceride levels significantly increased in the individuals with rs3480 GG genotype, and CKMB, total cholesterol, LDLc, troponin I, and triglyceridelevels significantly increased in the individual with rs726344 GA genotypes compared with other genotypes in patients with AMI. In the same study, they did not analyze the rs16835198 polymorphic variant in the FNDC5 gene. Associations between serum irisin level, CK-MB, and troponin $T$ were not detected in the FNDC5SNPs and cardiometabolic parameters in the present study. Our data indicated that the T allele and TT genotype of the rs16835198 SNP significantly decreased in male patients with $\mathrm{AMI}$, and there was no significant difference about to $A M I$, diabetes subgroup, and hypertension subgroup in the terms of other analyzed SNPs. In agreement with our results, Khidr et al. (15) demonstrated that the T allele and TT genotype of the rs16835198 SNP significantly decreased in patients with type II diabetes and diabetic nephropathy. We suggest that the T allele might contribute to the increased risk of disease develop- 
ment in male patients because male gender is a risk factor for the pathophysiology of AMI. A significant correlation was found between the rs16835298 and rs726344 SNPs and old age (9). In Japanese males, no correlation between cardiorespiratory exercise levels, SNPs of rs3480 and rs 16835198 in the FNDC5 gene, and serum irisin levels were found. The same study demonstrated that individuals with the $A G$ and $G G$ genotypes of rs3480 in the low exercise group had higher insulin and HOMAIR when compared with those with the AA genotypes (14). rs3480 variant was shown to form a binding region for mir-135a$5 P$, and this miRNA is increased in patients with Nonalcoholic fatty liver disease (NAFLD) and diabetic patients (16-19). In the present study, no significant difference was observed between the three analyzed SNPs' genotypes and serum irisin levels, consistent with the above-mentioned studies. Although a correlation was shown between rs3480 SNP and BMI in Saudi individuals with obesity, a similar correlation was not found between rs726344 and BMI (12). In investigations on effects of genetic polymorphisms, the differences in sample sizes, ethnicity, and populations can lead to the risk of false positive or negative findings. Therefore, the findings of the present study are needed to be corrected to be used in large study groups in the different populations.

\section{Study limitations}

The present study is not without its limitations. One of the most important limitations of our study is that the blood samples taken for CK-MB and troponin-T measurements were generally taken within the first hours after the patient presented to the emergency department, but the blood samples taken for irisin analysis were taken after the patients' hospitalization to the intensive care unit in the service. Future studies must focus on the measurement in the same blood samples of irisin, CK-MB, and troponin-T levels, especially to accurately evaluate the usefulness of irisin as a biomarker in the future. Second, we failed to obtain enough data, including the exercise condition of the volunteers. Thus, we cannot analyze the association of SNP or serum irisin levels and these data.

\section{Conclusion}

Our results demonstrated that the FNDC5 gene rs 16835198 G>T SNP provides significant protection against AMI in male patients without any effect on the serum irisin level. Consistent with CVD studies, it was demonstrated that serum irisin levels were significantly lower in patients with AMI. Although it is a highly promising molecule as a biomarker, extensive studies are needed to assess serum irisin levels both for the early diagnosis and the monitoring of $\mathrm{AMI}$ to evaluate its routine utility.

Acknowledgments: This study was sponsored by Firat University Research Foundation (Project No: TF.15.21).

Conflict of interest: None declared.

Peer-review: Externally peer-reviewed.
Author contributions: Concept - E.Ö.E., Ö.D.; Design - E.Ö.E., Ö.D.; Supervision - E.Ö.E.; Fundings - Firat University Research Foundation (Project No: TF.15.21); Materials - E.Ö.E., H.K.; Data collection \&/or processing - E.Ö.E., Ö.D., A.T., H.K., I.B.K.; Analysis \&/or interpretation - E.Ö.E.; Literature search - E.Ö.E., Ö.D., I.B.K.; Writing - E.Ö.E.; Critical review - E.Ö.E., I.B.K.

\section{References}

1. Murray CJ, Lopez AD. Alternative projections of mortality and disability by cause 1990-2020: Global Burden of Disease Study. Lancet 1997; 349: 1498-504. [Crossref]

2. Rodríguez A, Becerril S, Ezquerro S, Méndez-Giménez L, Frühbeck G. Crosstalk between adipokines and myokines in fat browning. Acta Physiol (Oxf) 2017; 219: 362-81. [Crossref]

3. Boström P, Wu J, Jedrychowski MP, Korde A, Ye L, Lo JC, et al. A PGC1- $\alpha$-dependent myokine that drives brown-fat-like development of white fat and thermogenesis. Nature 2012; 481: 463-8. [Crossref]

4. Roca-Rivada A, Castelao C, Senin LL, Landrove M0, Baltar J, Belén Crujeiras $A$, et al. FNDC5/irisin is not only a myokine but also an adipokine. PLoS One 2013; 8: e60563. [Crossref]

5. Sarioğlu G, Korkmaz H, Önalan E, Kuloğlu T, Yilmaz M, Özgüler M, et al. Irisin levels increase during experimentally induced acute myocardial infarction. Curr Res Cardiol 2016; 3: 9-12. [Crossref]

6. Aydin S, Aydin S, Kobat MA, Kalayci M, Eren MN, Yilmaz M, et al. Decreased saliva/serum irisin concentrations in the acute myocardial infarction promising for being a new candidate biomarker for diagnosis of this pathology. Peptides 2014; 56: 141-5. [Crossref]

7. Staiger H, Böhm A, Scheler M, Berti L, Machann J, Schick F, et al. Common genetic variation in the human FNDC5 locus, encoding the novel muscle-derived 'browning' factor irisin, determines insulin sensitivity. PLoS One 2013; 8: e61903. [Crossref]

8. Tang S, Zhang R, Jiang F, Wang J, Chen M, Peng D, et al. An interaction between a FNDC5 variant and obesity modulates glucose metabolism in a Chinese Han population. PLoS One 2014; 9: e109957. [Crossref]

9. Sanchis-Gomar F, Garatachea N, He ZH, Pareja-Galeano H, Fuku N, Tian $Y$, et al. FNDC5 (irisin) gene and exceptional longevity: a functional replication study with rs16835198 and rs726344 SNPs. Age (Dordr) 2014; 36: 9733. [Crossref]

10. Abdu Allah AM, Hammoudah SA, Abd El Gayed EM, El-Attar LM, Shehab-Eldin WA. Obesity and its Association with Irisin Level Among Individuals with FNDC5/Irisin Gene Variants RS16835198 and RS726344. Protein Pept Lett 2018; 25: 560-9. [Crossref]

11. Brondani LA, Boelter G, Assmann TS, Leitão CB, Canani LH, Crispim $D$. Irisin-encoding gene (FNDC5) variant is associated with changes in blood pressure and lipid profile in type 2 diabetic women but not in men. Metabolism 2015; 64: 952-7. [Crossref]

12. Al-Daghri NM, Mohammed AK, Al-Attas OS, Amer OE, Clerici M, Alenad A, et al. SNPs in FNDC5 (irisin) are associated with obesity and modulation of glucose and lipid metabolism in Saudi subjects. Lipids Health Dis 2016; 15: 54. [Crossref]

13. Ebert T, Kralisch S, Wurst U, Scholz M, Stumvoll M, Kovacs $P$, et al. Association of metabolic parameters and rs726344 in FNDC5 with serum irisin concentrations. Int $\mathrm{J}$ Obes (Lond) 2016; 40: 260-5. [Crossref]

14. Tanisawa K, Taniguchi H, Sun X, Ito T, Cao ZB, Sakamoto S, et al. Common single nucleotide polymorphisms in the FNDC5 gene are associated with glucose metabolism but do not affect serum irisin 
levels in Japanese men with low fitness levels. Metabolism 2014; 63 : 574-83. [Crossref]

15. Khidr EG, Ali SS, Elshafey MM, Fawzy OA. Association of irisin and FNDC5 rs16835198 G>T gene polymorphism with type 2 diabetes mellitus and diabetic nephropathy. An Egyptian pilot study. Gene 2017; 626: 26-31. [Crossref]

16. Metwally M, Bayoumi A, Romero-Gomez $M$, Thabet $K$, John $M$, Adams LA, et al. A polymorphism in the Irisin-encoding gene (FNDC5) associates with hepatic steatosis by differential miRNA binding to the 3'UTR. J Hepatol 2019; 70: 494-500. [Crossref]

17. He F, Peng F, Xia X, Zhao C, Luo Q, Guan W, et al. MiR-135a promotes renal fibrosis in diabetic nephropathy by regulating TRPC1. Diabetologia 2014; 57: 1726-36. [Crossref]

18. Agarwal P, Srivastava R, Srivastava AK, Ali S, Datta M. miR-135a targets IRS2 and regulates insulin signaling and glucose uptake in the diabetic gastrocnemius skeletal muscle. Biochim Biophys Acta 2013; 1832: 1294-303. [Crossref]

19. Sarookhani MR, Honardoost M, Foroughi F, Monfared YK. Plasma miR-135a; a potential biomarker for diagnosis of new type 2 diabetes (T2DM). Bali Med J 2018; 7: 296-301. [Crossref]

20. Shi YY, He L. SHEsis, a powerful software platform for analyses of linkage disequilibrium, haplotype construction, and genetic association at polymorphism loci. Cell Res 2005; 15: 97-8. [Crossref]

21. Reinehr T, Elfers $\mathrm{C}$, Lass $\mathrm{N}$, Roth $\mathrm{CL}$. Irisin and its relation to insulin resistance and puberty in obese children: a longitudinal analysis. $\mathrm{J}$ Clin Endocrinol Metab 2015; 100: 2123-30. [Crossref]

22. Anastasilakis AD, Polyzos SA, Saridakis ZG, Kynigopoulos G, Skouvaklidou EC, Molyvas D, et al. Circulating irisin in healthy, young individuals: day-night rhythm, effects of food intake and exercise, and associations with gender, physical activity, diet, and body composition. J Clin Endocrinol Metab 2014; 99: 3247-55. [Crossref]

23. Aronis KN, Moreno M, Polyzos SA, Moreno-Navarrete JM, Ricart W, Delgado $E$, et al. Circulating irisin levels and coronary heart disease: association with future acute coronary syndrome and major adverse cardiovascular events. Int J Obes (Lond) 2015; 39: 156-61. [Crossref]

24. Hsieh IC, Ho MY, Wen MS, Chen CC, Hsieh MJ, Lin CP, et al. Serum irisin levels are associated with adverse cardiovascular outcomes in patients with acute myocardial infarction. Int J Cardiol 2018; 261: 12-7. [Crossref]

25. Efe TH, Açar B, Ertem AG, Yayla KG, Algül E, Yayla Ç, et al. Serum Irisin Level Can Predict the Severity of Coronary Artery Disease in Patients with Stable Angina. Korean Circ J. 2017; 47: 44-9. [Crossref]
26. Kuloglu T, Aydin S, Eren MN, Yilmaz M, Sahin I, Kalayci M, et al. Irisin: a potentially candidate marker for myocardial infarction. Peptides 2014; 55: 85-91. [Crossref]

27. Mallet RT, Sun J. Mitochondrial metabolism of pyruvate is required for its enhancement of cardiac function and energetics. Cardiovasc Res 1999; 42: 149-61. [Crossref]

28. Aydin S, Kuloglu T, Aydin S, Kalayci M, Yilmaz M, Cakmak T, et al. A comprehensive immunohistochemical examination of the distribution of the fat-burning protein irisin in biological tissues. Peptides 2014; 61: 130-6. [Crossref]

29. Fan F, Sun A, Zhao H, Liu X, Zhang W, Jin X, et al. MicroRNA-34a promotes cardiomyocyte apoptosis post myocardial infarction through down-regulating aldehyde dehydrogenase 2. Curr Pharm Des 2013; 19: 4865-73. [Crossref]

30. Bernardo BC, Gao XM, Winbanks CE, Boey EJ, Tham YK, Kiriazis H, et al. Therapeutic inhibition of the miR-34 family attenuates pathological cardiac remodeling and improves heart function. Proc Natl Acad Sci U S A 2012; 109: 17615-20. [Crossref]

31. Köse SI, Maden M. Biomarkers and Clinical Usages. Dicle Üniv Vet Fak Derg 2013: 2: 30-7. [Article in Turkish]

32. Wen MS, Wang CY, Lin SL, Hung KC. Decrease in irisin in patients with chronic kidney disease. PLoS One 2013; 8: e64025. [Crossref]

33. Provatopoulou X, Georgiou GP, Kalogera E, Kalles V, Matiatou MA, Papapanagiotou $I$, et al. Serum irisin levels are lower in patients with breast cancer: association with disease diagnosis and tumor characteristics. BMC Cancer 2015; 15: 898. [Crossref]

34. Zhu H, Liu M, Zhang N, Pan H, Lin G, Li N, et al. Serum and Adipose Tissue mRNA Levels of ATF3 and FNDC5/Irisin in Colorectal Cancer Patients With or Without Obesity. Front Physiol 2018; 9: 1125. [Crossref]

35. Liu JJ, Wong MD, Toy WC, Tan CS, Liu S, Ng XW, et al. Lower circulating irisin is associated with type 2 diabetes mellitus. J Diabetes Complications 2013; 27: 365-9. [Crossref]

36. Zhang W, Chang L, Zhang C, Zhang R, Li Z, Chai B, et al. Central and peripheral irisin differentially regulate blood pressure. Cardiovasc Drugs Ther 2015; 29: 121-7. [Crossref]

37. Badr EA, Mostafa RG, Awad SM, Marwan H, Abd El-Bary HM, Shehab $\mathrm{HE}$, et al. A pilot study on the relation between irisin singlenucleotide polymorphism and risk of myocardial infarction. Biochem Biophys Rep 2020; 22: 100742. [Crossref] 